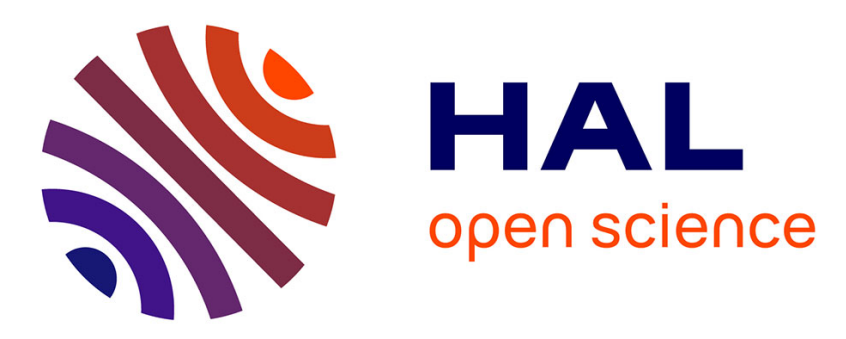

\title{
Does roughening of rock-fluid-rock interfaces emerge from a stress-induced instability?
}

Eric Bonnetier, Chaouqi Misbah, François Renard, Renaud Toussaint, Jean Pierre Gratier

\section{To cite this version:}

Eric Bonnetier, Chaouqi Misbah, François Renard, Renaud Toussaint, Jean Pierre Gratier. Does roughening of rock-fluid-rock interfaces emerge from a stress-induced instability?. The European Physical Journal B: Condensed Matter and Complex Systems, 2009, 67 (1), pp.121-131. 10.1140/epjb/e2009-00002-2 . insu-00352886

\section{HAL Id: insu-00352886 https://hal-insu.archives-ouvertes.fr/insu-00352886}

Submitted on 14 Jan 2009

HAL is a multi-disciplinary open access archive for the deposit and dissemination of scientific research documents, whether they are published or not. The documents may come from teaching and research institutions in France or abroad, or from public or private research centers.
L'archive ouverte pluridisciplinaire HAL, est destinée au dépôt et à la diffusion de documents scientifiques de niveau recherche, publiés ou non, émanant des établissements d'enseignement et de recherche français ou étrangers, des laboratoires publics ou privés. 


\title{
Does roughening of rock-fluid-rock interfaces emerge from a stress-induced instability?
}

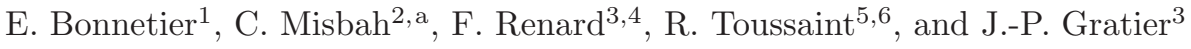 \\ ${ }^{1}$ Laboratoire Jean Kuntzmann, Université Joseph Fourier and CNRS, B.P. 53, 38041 Grenoble Cedex 9, France \\ 2 Laboratoire de Spectrométrie Physique, 140 avenue de la physique, Université Joseph Fourier, and CNRS, 38402, Saint Martin \\ d'Hères, France \\ 3 Laboratoire de Géodynamique des Chaines Alpines, CNRS-OSUG, Université Joseph Fourier, B.P. 53, 38041 Grenoble, France \\ 4 Physics of Geological Processes, University of Oslo, Norway \\ ${ }^{5}$ Institut de Physique du Globe de Strasbourg, UMR CNRS 7516, 5 rue Descartes, 67084 Strasbourg Cedex, France \\ ${ }^{6}$ EOST, Université de Strasbourg, France
}

Received 20 October 2008 / Received in final form 24 November 2008

Published online (Inserted Later) - C) EDP Sciences, Società Italiana di Fisica, Springer-Verlag 2008

\begin{abstract}
Non-planar solid-fluid-solid interfaces under stress are very common in many industrial and natural materials. For example, in the Earth's crust, many rough and wavy interfaces can be observed in rocks in a wide range of spatial scales, from undulate grain boundaries at the micrometer scale, to stylolite dissolution planes at the meter scale. It is proposed here that these initially flat solid-fluid-solid interfaces become rough by a morphological instability triggered by elastic stress. A model for the formation of these unstable patterns at all scales is thus presented. It is shown that such instability is inherently present due to the uniaxial stress that promotes them, owing to the gain in the total elastic energy: the intrinsic elastic energy plus the work of the external forces. This is shown explicitly by solving the elastic problem in a linear stability analysis, and proved more generally without having resort to the computation of the elastic field.
\end{abstract}

PACS. 91.32.De Crust and lithosphere - 68.35.Fx Diffusion; interface formation - 02.30.Jr Partial differential equations - Plasticity, diffusion, and creep Plasticity, diffusion, and creep - 91.60.Dc Plasticity, diffusion, and creep

\section{Introduction}

When a solid is non-uniformly loaded (Fig. 1), its elastic free energy is increased and local gradients of freeenergy can induce mass transfer from the most stressed sides of the solid to the least stressed ones, or to other surrounding solids, to minimize the energy increase related to the loading. The interface kinetics of the stressed solid is controlled by the slowest mechanism by which the mass is transported. This configuration is found in many layered industrial materials or natural systems. For example, in the rocks of the Earth's crust, loaded interfaces are widespread: fault surfaces and stylolites (Fig. 2) at a macroscopic scale; grain boundaries and grain free surfaces in a porous medium at the microscopic scale.

Two different geometries can be defined, depending on the orientation of the main compressive stress relative to the loaded interface (Fig. 1).

\footnotetext{
a e-mail: chaouqi.misbah@ujf-grenoble.fr
}

- When the main compressive stress is parallel to the surface, grooves can develop, this is the Asaro-Tiller-Grinfeld instability [3,11,12,21,23,31], referred to, later in this paper, as "the free-face instability". This instability is well understood theoretically. It has been observed on Helium by Torii and Balibar [33]. It has also been proposed that it could be reproduced experimentally on sodium chlorate single crystals [7]. However, experiments on the same salt do also show that this instability may disappear after some time. This effect might be related to the precipitation of a stress-free skin at the surface of the crystals [5].

- When the main compressive stress is perpendicular to the solid surface, initially flat dissolution surfaces can become rough in the course of time by a dissolution process. Typical natural examples of such squeezed unstable interfaces can be observed in natural rocks. They are called stylolites (Fig. 2). In sedimentary basins, stylolites are observed as rough horizontal interfaces $[8,13,24,30,32]$. There, the main compressive 
stress is vertical and corresponds to the weight of the overburden rocks. In mountain chains, where the main compressive stress corresponds to the horizontal tectonic loading, rough stylolite surfaces are oriented vertically $[2,25]$. From these basic observations, one may conclude that stress is a key ingredient in stylolite pattern formation $[4,17]$. In the present study, we call such roughening process "the squeezed interface instability". It differs from the free-face instability by the orientation of the main compressive stress. This second instability is less understood. It has been proposed that the roughening of the interface is controlled by a destabilizing force, the noise initially present in the rock [26,27]. In [9] it was assumed that diffusion occurs along the solid-solid interface and a simple model to describe the instability has been proposed. However, a model that takes into account a more realistic geometry is lacking, together with a systematic derivation of the governing equations. Furthermore, it remains to be shown whether or not a purely elastic instability explains the formation of stylolites. This paper is directed along these lines.

We present a model that shows that squeezed solid-fluidsolid interfaces are unstable due to stress. This situation is less classical than the one usually treated: here two solids are in contact with a thin liquid layer and the weight is transmitted from one solid to the other by the liquid layer. It is thus essential to derive the equations and boundary conditions in this geometry. We must take into account not only the intrinsic elastic energy but also the work due to external forces.

Natural and experimental observations of rough surfaces indicate that stress has a strong control on the evolution of the fluid-solid interfaces: stress gradients are released by dissolution-precipitation or melting solidification processes, which modify the solid texture and induce irreversible deformations (see Fig. 2).

The scheme of this paper is as follows. In Section 2 we briefly review the free interface case. In Section 3 we treat the squeezed interface case by performing a linear stability analysis, and present the main results that reveals an instability driven by stress. In Section 4 we present a more general and formal proof of the instability without having resort to an explicit solution of the elastic field. Section 5 is devoted to a general discussion. Some technical details are presented in an appendix.

\section{The free interface case}

If the main compressive stress is parallel to the loaded interface (Fig. 1a), grooves can develop on the free surface. This is the well-known Asaro-Tiller-Grinfeld instability $[3,11]$. It has been found experimentally $[7,19,33]$ that the formation of the grooves occurs on a free surface of various solids in contact with a fluid when a load (or a uniaxial stress [33]) is applied. The grooves can theoretically evolve to fractures that propagate at a subcritical rate $[14,16,18,31,34]$. The wavelength of the instability

\section{free surface instability}

a)

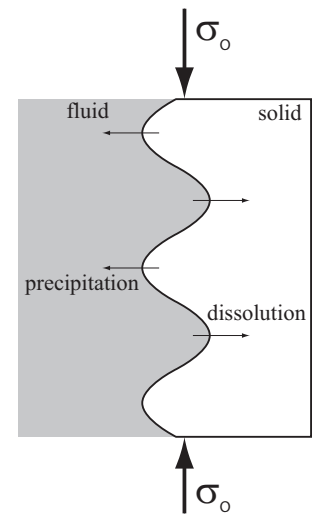

stylolite instability

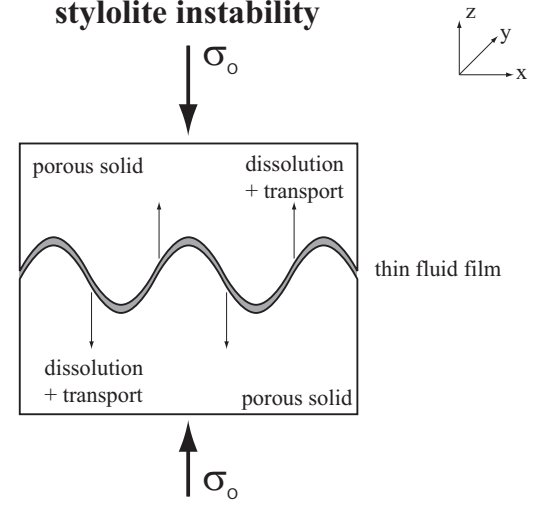

Fig. 1. Morphological instabilities of a solid-fluid interface and effect of the orientation of the main compressive stress. (a) Free-face instability: when a free surface of a solid in contact with a fluid is loaded perpendicular to the surface, grooves can develop through time and even evolve to cracks. This is the Asaro-Tiller-Grinfeld instability. (b) Squeezed interface instability: A typical example is a stylolite, which corresponds to a fluid-filled rock-rock interface loaded perpendicularly to the interface. The mean roughness amplitude of the interface grows with time, which gives their characteristic shapes to the stylolites. In both cases, the fluid phase acts as a reactive medium transporting solutes by diffusion and allowing stress driven dissolution-precipitation processes at the interface with the solid.

is controlled by a balance between elastic forces, which tend to roughen the surface, and surface tension, which smoothen it. The characteristic wavelength $\lambda_{c}$ of the instability that emerges from a linear stability analysis is

$$
\lambda_{c}=\frac{\pi E \gamma}{\sigma_{0}^{2}\left(1-\nu^{2}\right)},
$$

where $E$ is the Young modulus of the solid, $\gamma$ is the interfacial energy between the solid and the liquid, $\nu$ is the Poisson coefficient, and $\sigma_{0}$ is the applied main compressive stress (see Fig. 1). The planar front is unstable if the perturbation wavelength $\lambda$ is such that $\lambda>\lambda_{c}$ and it is stable otherwise. 
a)
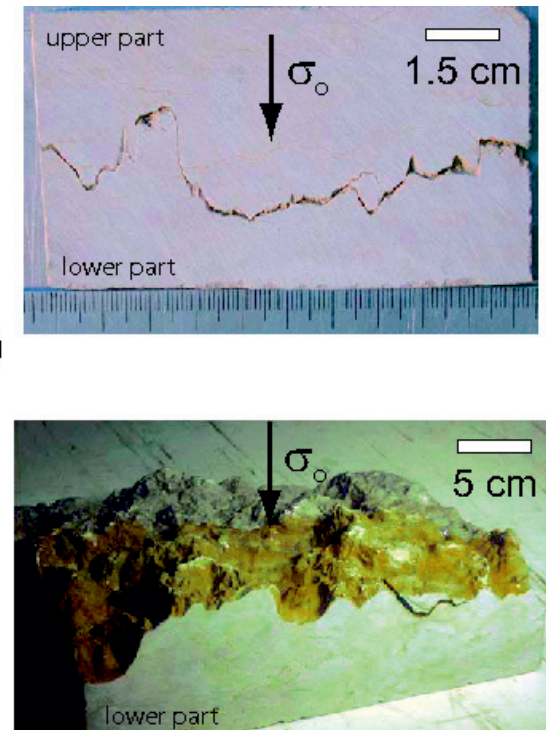

b)

d)

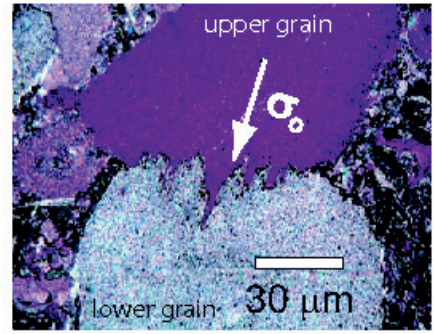

C)

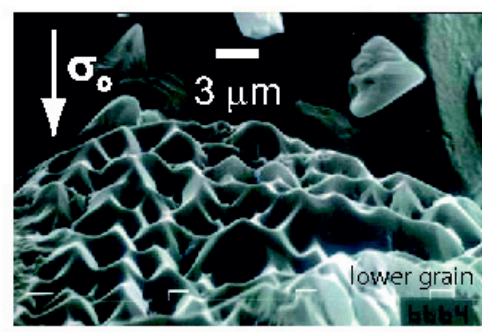

Fig. 2. Various patterns of rough stylolite interfaces at all scales. (a) Stylolite interface in a sedimentary limestone from the Chartreuse Mountains, France. The rough interface pattern formed by stress-enhanced dissolution from an initially flat rockfluid-rock interface. In this case, the maximum compressive stress $\sigma_{0}$ was perpendicular to the interface. (b) Stylolite surface viewed in 3D after removing the upper part of a limestone, similar to the sample shown in (a). (c) Microscopic observation of a rough grain-grain boundary in a limestone from Mons, Belgium, showing two spherical grains indented into each other. The rough teeth pattern of the interface has formed by a stress-enhanced dissolution process. (d) Scanning Electron Microscope view of a quartz grain surface, after experimentally produced stress-enhanced dissolution against a second quartz grain that has been removed for better visualization. The maximum stress was vertical, and perpendicular to the rough interface. Adapted from $[10]$.

For the case of rocks, in which we are interested here, it has been shown that the transport mechanism may be controlled either by dissolution kinetics of the crystal, or diffusion of solutes in the fluid $[9,21]$. This depends on the nature of rocks, as discussed in [21].

\section{The squeezed interface case}

\subsection{Presentation of the instability}

Consider the situation depicted in Figure 3, where the initial surface representing the position of the squeezed interface $\Gamma$ is set at $z=0$. The interface contains a thin water film at a pressure $p$, squeezed between the two solids that have identical linear elastic properties. We consider, for the sake of simplicity, one dimensional deformations along $x$ only, so that the stress and the strain fields are independent of $y$. Here we shall not describe the mechanisms by which the modulation takes place, but, rather, we are interested to compare the energetic of the initial state (flat) with that of a corrugated one.

Due to the assumption of translational invariance along $y$, the problem reduces to an effective $2 \mathrm{D}$ one where it is convenient to make use of the Airy function $\chi(x, z)$ which is defined in terms of the stress tensor as [20]:

$$
\sigma_{x x}=\frac{\partial^{2} \chi}{\partial z^{2}}, \sigma_{z z}=\frac{\partial^{2} \chi}{\partial x^{2}}, \sigma_{x z}=-\frac{\partial^{2} \chi}{\partial x \partial z} .
$$

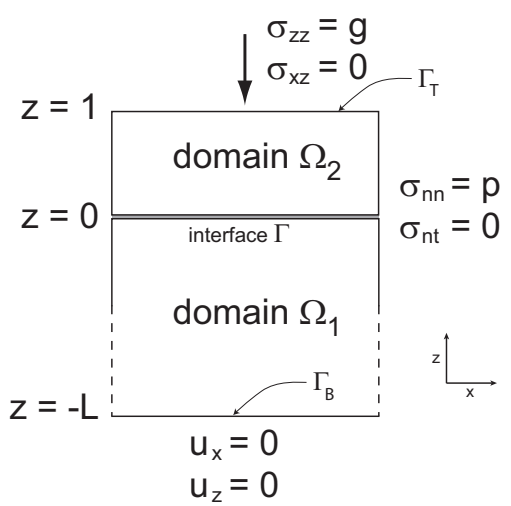

Fig. 3. Geometry of the system: two linear elastic solids with identical moduli are pushed into each other and separated by a thin confined water film at pressure $p$. The boundaries conditions used in Sections 3 and 4 are given.

The Airy function $\chi$ obeys a bi-harmonic equation [20]:

$$
\nabla^{4} \chi=0
$$

Once $\chi$ is determined the stress can be computed from the very definition of $\chi$, and the strain is obtained from Hooke's law. It must be emphasized that since we confine ourselves to two dimensional deformations, the strainstress relation differs from the three dimensional version (as far as the coefficients are concerned). We have in two 
dimensions the relations

$$
\begin{aligned}
& \varepsilon_{x x}=\frac{1+\nu}{E}\left[(1-\nu) \sigma_{x x}-\nu \sigma_{z z}\right] \\
& \varepsilon_{z z}=\frac{1+\nu}{E}\left[(1-\nu) \sigma_{z z}-\nu \sigma_{x x}\right] \\
& \varepsilon_{x z}=\frac{1+\nu}{E} \sigma_{x z}
\end{aligned}
$$

2 where $E$ is the Young's modulus of the solid and $\nu$ is the Poisson's ratio. This limit is also known as the plane strain condition.

The interface equation is written as $z=h(x)$. For $h=0, \chi_{0}=g x^{2} / 2$ (the subscript ' 0 ' refers to the planar interface) is obviously a solution of (3) with $\sigma_{z z}=g$ the only non zero component. This solution satisfies the boundary condition at $z=1$, and from $\sigma_{n n}=p$, we obtain $p=g$. This is physically appealing since the equilibrium at the interface, where the force is normal and equal to $p$, requires a compensation of the applied load $g$. Apart from a hydrostatic pressure (taken as an origin), both $\sigma_{x x}=0$ and $\sigma_{x z}=0$. The pre-strained situation is uniaxial.

Let us now assume that the interface undergoes a virtual displacement $h(x)$ and compute the resulting elastic fields in both solid domains denoted as ' 1 ' and ' 2 '. Of course in its great generality this problem is highly nonlinear for an arbitrary $h$, and can only be dealt with numerically. If one is interested in determining whether or not an interface displacement results in a gain of energy, it may be sufficient to perform a linear stability analysis, a problem which can ba handled analytically.

Because different modes do not interact in the linear regime, it is sufficient to consider only one Fourier component, namely we seek solutions in the form:

$$
h=\varsigma e^{i q x}+c . c .
$$

In domain ' 2 ' we have

$$
f=\left(A_{2} z+B_{2}\right) e^{q z}+\left(A_{2}^{\prime} z+B_{2}^{\prime}\right) e^{-q z} .
$$

where $f$ is a function which is yet unknown. From (3) it follows that $f$ obeys

$$
\left[\frac{\partial^{2}}{\partial z^{2}}-q^{2}\right]^{2} f=0
$$

The eight integration factors are determined by the eight conditions: the normal stress at $z=1$ is equal to $g$ where the surface there is free from shear. These two conditions read

$$
\left.\sigma_{z z}\right|_{z=1}=g,\left.\quad \sigma_{x z}\right|_{z=1}=0 .
$$

At the interface the normal components on both sides coincide with $p$, while the tangential components vanish. This amounts to four independent conditions

$$
\begin{aligned}
& \left.\sigma_{n n}\right|_{z=0^{-}}=p,\left.\quad \sigma_{n n}\right|_{z=0^{+}}=p, \\
& \left.\sigma_{n t}\right|_{z=0^{-}}=0,\left.\quad \sigma_{n t}\right|_{z=0^{-}}=0
\end{aligned}
$$

where $\sigma_{n n}=n_{i} \sigma_{i j} n_{j}$ and $\sigma_{n t}=n_{i} \sigma_{i j} t_{j}$, with $n_{i}$ and $t_{j}$ representing the $i^{t h}$ component of the normal and the tangent vectors evaluated at the interface. Note that from zeroth order solution we have seen that $p=g$, so that from now on we shall abandon the $p$ symbol in favor of $g$.

Finally at the bottom, $z=-L$, we impose a zero displacement condition, namely

$$
\left.u_{z}\right|_{z=-L}=0,\left.u_{x}\right|_{z=-L}=0 .
$$

Using the Airy function and the definition (2) together with (9) and (10), and expanding the equations to order one in $\varsigma$ we obtain eight equations determining the eight unknowns. The solutions take the form

$$
\begin{aligned}
& A_{1}=g\left[e^{-2 q L}(2 q L+1)+3-4 \nu\right] / D_{1} \\
& B_{1}=2 g e^{-2 q L}\left[q^{2} L^{2}+2+4 \nu^{2}-6 \nu\right] / D_{1} \\
& A_{1}^{\prime}=g e^{-2 q L}\left[1-2 q L+e^{-2 q L}(3-4 \nu)\right] / D_{1} \\
& B_{1}^{\prime}=-B_{1} \\
& A_{2}=-g\left[e^{2 q}(2 q-1)+1\right] / D_{2} \\
& B_{2}=2 q g e^{2 q} / D_{2} \\
& A_{2}^{\prime}=-g e^{2 q}\left[e^{2 q}-2 q-1\right] / D_{2} \\
& B_{2}^{\prime}=-2 q g e^{2 q} / D_{2}
\end{aligned}
$$

where we have set

$D_{1}=(3-4 \nu)\left[1+e^{-4 q L}\right]+2 e^{-2 q L}\left[2 q^{2} L^{2}+8 \nu^{2}-12 \nu+5\right]$

and

$$
D_{2}=2 e^{2 q}\left(2 q^{2}+1\right)-1-e^{4 q} .
$$
the elastic field can be obtained straightforwardly.

\subsection{Energy considerations}

In this section we will be mainly concerned with the total energy of the system in the deformed and undeformed states. The total energy should contain both the intrinsic part and the work of the external force, $g$. The energy contribution from the intrinsic part is $1 / 2 \int_{\Omega} \sigma d \tau: \varepsilon(u)$ where $\Omega$ is the total volume, and the work of the external force is $-\int_{\Gamma_{T}} g u$, with $\Gamma_{T}$ is the upper boundary (see Fig. 3). The total energy is thus

$$
E=\frac{1}{2} \int_{\Omega} \sigma: \varepsilon(u) d \tau-\int_{\Gamma_{T}} g u .
$$


It will be shown in Section 4 that minimization of this energy with respect to $u$ yields the appropriate elastic equations, $\operatorname{div}(\sigma)=0$ and the boundary conditions (11), (12), and (13). Upon substitution of the equilibrium condition, the relaxed elastic energy will then take the following form

$$
E_{0}=-\frac{1}{2} \int_{\Omega} \sigma: \varepsilon(u) d \tau
$$

where the subscript ' 0 ' is to remind us that the quantity under consideration is the relaxed energy. That this quantity is negative is obvious, since the relaxed energy should be smaller than the non-relaxed one, otherwise there is a trivial solution which would have a zero energy, the one corresponding to a zero displacement.

It remains now to be shown that the variation of this quantity with respect to an interface modulation $\varsigma \cos (q x)$ (produced due to some mass transport) is negative, a signature of the instability. A general proof is presented in Section 4 without resorting to the explicit form of the elastic field. It is also of interest to have an explicit expression of the energy, (and possibly of the chemical potential), if one wishes to study the kinetics of the instability, and provide the appropriate length and time scales of the evolution.

We should remind that $\sigma$ has a zeroth order contribution due to pre-strain, and in computing the energy $E_{0}$ one has to subtract the energy of the pre-strained state, so that the obtained form contains the contribution due to the profile $z=h(x, t)$. In the linear regime of perturbation with respect to $h$ (i.e. the stress is computed up to order $h$ ), the energy $E_{0}$ assumes a quadratic form. In the general situation where the extent of the upper and lower parts of the sample is finite the energy is lengthy enough so we did not feel it worthwhile to list it here. We give only the limit where $q L \gg 1$ (lower part, below the interface, is large in comparison to lengths of interest):

$$
\begin{aligned}
E_{0}= & q g^{2} \frac{1+\nu}{E D_{2}}\left\{(1-2 \nu)\left[1+e^{4 q}-2 e^{2 q}-4 q^{2} e^{2 q}\right]\right. \\
& \left.-4 q e^{2 q}+e^{4 q}-1\right\} \varsigma^{2}
\end{aligned}
$$

where $D_{2}$ is a constant defined in equation (16). The above energy is computed per unit period along $x$ and per unit length along $y$. If the surface energy (39) is taken into account, one has to supplement $E_{0}$ with the following contribution

$$
E_{s}=\gamma \int d x\left(\frac{d h}{d x}\right)^{2}
$$

the relaxed energy would be the opposite of (18), and therefore $E_{0}$ would have been positive in equation (19), signaling a stability instead of instability. This will further be shown in the general treatment in Section 4. In contrast to elasticity, the surface energy is stabilizing. A remark is in order. The comparison of the elastic energy (which is destabilizing) and the surface energy (which is stabilizing) has also a similar spirit as that due Griffith in fracture theory. Indeed, in Griffith theory a crack propagates if its length $\ell$ exceeds a typical value given by the ratio of the loss of surface energy $\gamma$ over the gain in elastic energy (crack releases stored elastic energy) $\sim \sigma_{0}^{2} / E$. More interesting is that the Griffith condition, according to which a crack propagates when its length exceeds a critical length $\ell_{c}$, is precisely (apart from a numerical factor of order unity) the condition of the ATG instability: the planar front is unstable if the wavelength is larger than $\lambda_{c}$ (see Eq. (1)), whereas the Griffith condition states [20] that a crack propagates if its length $\ell>\ell_{c}=\left(4 / \pi^{2}\right) \lambda_{c}$.

We shall first discuss the two extreme limits of large and short wavenumbers. In these extreme limits the expression takes a very simple form. The first case is, perhaps, the most relevant one for natural systems such as stylolites, where we assume that $q \gg 1$ (short wavelength). This means that we take the limit where the modulation wavelength is small as compared to the interface extent. The energy (per unit period) takes then the form

$$
E_{0}=-\frac{4\left(1-\nu^{2}\right)}{E}|q| g^{2} \varsigma^{2}
$$

which is negative, signaling a morphological instability. Note that we keep $|q|$ in the expression above in order to stress the nonlocal character of the elastic field. Indeed, in real space the quantity $|q|$ leads to a Hilbert transform of $\partial_{x} h(x)$. More precisely

$$
T F^{-1}\left(|q| h_{q}\right)=(1 / \pi) \mathcal{P} \int \frac{\partial_{x^{\prime}} h\left(x^{\prime}\right)}{x^{\prime}-x},
$$

where $T F$ stands for a Fourier transform, and $h_{q}=$ $T F^{-1}(h)\left(T F^{-1}\right.$ designates the inverse Fourier transform). The symbol $\mathcal{P}$ refers to the fact that the integral must be taken in the sense of the Cauchy principal value. For a real function $f(x)$ the Cauchy principal value is defined as

$$
\mathcal{P} \int_{-\infty}^{\infty} \frac{f(x)}{x} d x \equiv \lim _{\epsilon \rightarrow 0}\left[\int_{-\infty}^{-\epsilon} \frac{f(x)}{x} d x+\int_{\epsilon}^{\infty} \frac{f(x)}{x} d x\right] .
$$

Let us abbreviate this expression as $p v(f(x) / x)$. Applying $T F$ on both sides of (23), one gets on the left hand side $|q| h_{q}$, while the right hand side is a convolution providing a product of $T F\left(\partial_{x^{\prime}} h\left(x^{\prime}\right)\right)$ and $T F\left(p v\left(1 /\left(x^{\prime}-x\right)\right)\right.$. The first term yields $i q h_{q}$, while the second one is equal to $-i \pi \operatorname{sgn}(q)$ (a classical result of theory of distributions, and can easily be obtained by using the residue theorem), $\operatorname{sgn}(q)$ stands for 'sign of $q$ '. The final result (after accounting for the factor $\pi$ in (23)) is $q \operatorname{sgn}(q) h_{q}=|q| h_{q}$, that is identical to the left hand side result.

46 is always negative, signaling an instability. Note that if the

47 work of the external forces in (17) is not included, then 
In the opposite limit $(q \ll 1)$ one gets (for $L=1$ )

$$
E_{0}=-\frac{2(1+\nu)}{15 E(1-\nu)} g^{2} q^{2}(17-32 \nu) \varsigma^{2}
$$

The effect of the confinement leads to a spectrum which begins with $q^{2}$ instead of $q$. This may have, in principle, some significant consequences, as discussed below.

For example, in the non-confined regime the elastic energy (which is $\sim q$ ) dominates at small $q$ in comparison to the surface energy which behaves as $q^{2}$. This means that the instability is always present there. The typical lengthscale of the instability is given by balancing the elastic energy $\sim q g^{2} / E$ with the surface energy $\sim \gamma q^{2}$ where $\gamma$ is the surface energy. This leads to a typical length scale $\lambda_{c} \sim E \gamma / g^{2}$.

In the confined regime the energy behaves as $-q^{2} g^{2} \ell E$ (where for homogeneity reasons we have reintroduced a length scale $\ell$ representing a typical length of the vertical extent of the solid), precisely like the surface energy regarding the $q$ dependence, $+q^{2} \gamma$. Since the latter is stabilizing, while the former is destabilizing, an instability may take place only if $\ell>\gamma E / g^{2} \cdot g$ has a dimension of $E$ and can be written as $g=n E$ where $n$ is a dimensionless number smaller than one. We must have then $\ell>\gamma /(n E)$. In most cases $\gamma / E$ is of the order of an atomic length and $n$ is small enough so we conclude that for all practical purposes the instability takes place.

\section{A general framework}

In this section, we cast the previous calculation in the framework of a variational analysis. We provide a rigorous mathematical derivation on the stress-induced instability. Unlike the previous derivation, the present result will be obtained without knowing the explicit expression of the elastic field. We restrict ourselves to a $2 \mathrm{D}$ situation for simplicity; however, the analysis carries over to the 3D case. We consider a configuration similar to that of Figure 3: a portion of interface $\Gamma$ separates two pieces of solids $\Omega_{1}$ and $\Omega_{2}$ in the rectangle $\Omega=\Omega_{1} \cup \Omega_{2} \cup \Gamma$.

\subsection{Mechanical equilibrium for a fixed interface $\Gamma$}

We view the rectangle $\Omega=(0,1) \times(0,1)$ as a small slab of solid around an interface and assume periodic boundary conditions on the vertical sides $\Gamma_{V}=\{0\} \times(0,1) \cup$ $\{1\} \times(0,1)$. We assume that $\Omega_{1}$ lies below $\Omega_{2}$ and that both are sufficiently regular open sets (say with Lipschitz boundaries). A vertical load with modulus $g$ is applied to the top boundary $\Gamma_{T}$ and the displacement $u_{1}$ is fixed on the bottom boundary $\Gamma_{B}$. We use the Einstein summation convention of repeated indices.

The transmission conditions between $\Omega_{1}$ and $\Omega_{2} \bmod -$ els the presence of a very thin layer of fluid in the interface. We assume therefore that the stress tensors $\sigma_{1}$ and $\sigma_{2}$ in $\Omega_{1}$ and $\Omega_{2}$ satisfy

$$
\sigma_{i} n_{i}=p n_{i}, \quad \text { on } \Gamma, i=1,2,
$$

where $n_{i}$ denotes the outward normal to $\Omega_{i}$, and where $p$ is the Lagrange multiplier that denotes the (unknown) pressure in the thin layer of fluid. Altogether, the mechanical equilibrium of the system is expressed by the equations

$$
\left\{\begin{array}{cl}
-\operatorname{div}\left(\sigma_{i}\right) & =0 \quad \text { in } \Omega_{i} \\
\sigma_{i} & =A \varepsilon\left(u_{i}\right) \quad \text { in } \Omega_{i}, \\
\sigma_{2} n_{2} & =g n_{2} \text { on } \Gamma_{T} \\
u_{1} & =0 \text { on } \Gamma_{B} \\
u_{i} & \text { periodic on } \Gamma_{V}, \\
\sigma_{i} n_{i} & =\text { pn } n_{i} \text { on } \Gamma
\end{array}\right.
$$

where $i=1,2, \varepsilon(u)=1 / 2\left(\nabla u+\nabla u^{T}\right)$ is the symmetric strain tensor, and $A$ is the $4 \times 4$ tensor of isotropic Lamé coefficients of the solid. Alternatively, the above partial differential equations can be obtained as the Euler Lagrange equations of the following energy functional

$$
E_{\Gamma}\left(v_{1}, v_{2}\right)=\frac{1}{2} \int_{\Omega_{i}} A \varepsilon\left(v_{i}\right): \varepsilon\left(v_{i}\right) d x-\int_{\Gamma_{T}} g n_{2} v_{2} .
$$

The set $V$ of admissible displacements $V_{\Gamma}$ consists of pairs $\left(v_{1}, v_{2}\right): \Omega_{1} \times \Omega_{2} \longrightarrow \mathbf{R}^{2} \times \mathbf{R}^{2}$ of square integrable functions, with square integrable derivatives, such that

$$
\left\{\begin{array}{c}
v_{1}=0 \text { on } \Gamma_{T} \\
v_{1}, v_{2} \text { periodic on } \Gamma_{V} \\
\int_{\Gamma} v_{1} n_{1}+v_{2} n_{2}=0
\end{array}\right.
$$

Note that the constraint on the normal displacements on $\Gamma$ is associated with the Lagrange multiplier $p$ introduced above. One easily checks that minimizing $E_{\Gamma}$ over $V_{\Gamma}$ yields a solution $\left(u_{1}, u_{2}\right)$ to the corresponding Euler-Lagrange equation (26), which is defined up to a horizontal translation of $u_{2}$. To obtain a well-defined solution we further impose the normalization condition

$$
\int_{\Gamma_{T}} u_{2}\left(\begin{array}{l}
1 \\
0
\end{array}\right)=0 .
$$

\subsection{First variation with respect to the interface $\Gamma$}

In this paragraph, we compute the shape derivative, with respect to variations of the interface $\Gamma$, of the elastic energy functional

$$
J(\Gamma)=\min _{\left(v_{1}, v_{2}\right) \in V(\Gamma)} E_{\Gamma}\left(v_{1}, v_{2}\right) .
$$

Denoting $\left(u_{1}, u_{2}\right)$ the solution of the above variational problem (the actual elastic displacements for the geometry defined by the interface $\Gamma$, under the loading $g$ ) using (26), and integrating by parts shows that

$$
\begin{aligned}
J(\Gamma) & =\frac{1}{2} \int_{\Omega_{i}} A \varepsilon\left(u_{i}\right): \varepsilon\left(u_{i}\right) d x-\int_{\Gamma_{T}} g n_{2} u_{2} \\
& =-\frac{1}{2} \int_{\Omega_{i}} A \varepsilon\left(u_{i}\right): \varepsilon\left(u_{i}\right) \\
& =-\frac{1}{2} \int_{\Gamma_{T}} g n_{2} u_{2} .
\end{aligned}
$$


To differentiate the functional with respect to variations of the shape of $\Gamma$, we follow the approach of Murat and Simon $[22,28]$ which we now briefly recall: Consider perturbations of an open set $\omega \subset \mathbf{R}^{2}$ of the form

$$
\omega_{t}=\omega+t \theta
$$

where $\theta: \mathbf{R}^{2} \longrightarrow \mathbf{R}^{2}$ is a sufficiently smooth function, and $t$ is a small real parameter (the limit $t \rightarrow 0$ will be taken eventually).

Let $z$ be a smooth function and consider the functionals, defined respectively as a volume integral and a surface integral

$$
\begin{aligned}
J_{1}^{\prime}(\omega) \theta= & \int_{\omega} \partial_{u} z(u) u^{\prime}+\int_{\partial \omega} z(u) \theta n \\
J_{2}^{\prime}(\omega) \theta= & \int_{\partial \omega} \partial_{u} z(u) u^{\prime} \\
& +\int_{\partial \omega}\left[H z(u)+\partial_{n} z(u)\right] \theta n
\end{aligned}
$$

where $\partial_{n} f(x)=\nabla f(x) n$ is the normal derivative of $f$. The presence of the mean curvature $H$ on $\partial \omega$ in the derivative of the surface integral $J_{2}$ results from taking variations of the surface measure. In these expressions, the local derivative $u^{\prime}$ of $u$ at $x \in \omega$ is defined by

$$
u^{\prime}(x)=\lim _{t \rightarrow 0} \frac{u_{t}(x)-u(x)}{t},
$$

where $u_{t}$ is the solution to $A u=0$ in $\omega_{t}$ with the boundary conditions $B_{t} u_{t}=0$.

In our context, we consider perturbations $\left(\Omega_{1}, \Omega_{2}\right)$ of the form

$$
\Omega_{i}^{t}=\Omega_{i}+t \theta(x, y), \quad i=1,2,
$$

where $\theta: \mathbf{R}^{2} \longrightarrow \mathbf{R}^{2}$ is sufficiently smooth. We assume that $\theta$ leaves the outer boundary $\partial \Omega$ fixed, (i.e., $\theta$ only modifies the shape of the interface) and that it preserves which imposes that the volume of each subdomain $\Omega_{i}$

$$
\left\{\begin{array}{l}
\theta(x, y)=0 \quad \text { on } \partial \Omega \\
\left|\Omega_{i}^{t}\right|=\left|\Omega_{i}\right| \quad i=1,2
\end{array}\right.
$$

$$
\int_{\Gamma} \theta n_{i}=0
$$

Let $\left(u_{1}^{t}, u_{2}^{t}\right)$ denote the solution to (26) for the configuration $\Omega_{t}$

$$
\left\{\begin{array}{rl}
\operatorname{div}\left(A e\left(u_{i}^{t}\right)\right) & =0 \quad \text { in } \Omega_{i}^{t} \\
u_{1}^{t} & =0 \text { on } \partial \Omega_{i} \cap \Gamma_{B} \\
A \nabla u_{2}^{t} n_{2} & =g n_{2} \text { on } \Gamma_{T} \\
u_{i}^{t} & \text { periodic on } \Gamma_{V} \\
A e\left(u_{i}^{t}\right) n_{i}^{t} & =p_{t} n_{i}^{t}, \quad \text { on } \Gamma+t \theta .
\end{array} \quad i=1,2 .\right.
$$

The local derivatives $\left(u_{1}^{\prime}, u_{2}^{\prime}\right)$ satisfy

$$
\operatorname{div}\left(A e\left(u_{i}^{\prime}\right)\right)=0 \quad \text { in } \Omega_{i}
$$

and are periodic on the sides $\Gamma_{V}$. The boundary condition (26.d) implies that $u_{1}^{\prime}+\theta_{n} \partial_{n} u_{1}=0$ on $\Gamma_{B}$, which, given the hypothesis on $\theta$, reduces to

$$
u_{1}^{\prime}=0 \quad \text { on } \Gamma_{B} .
$$

In the Appendix, we derive the expression of the shape derivative of $J(\Gamma)$. If $\Gamma \subset(0,1) \times(0,1)$ is a periodic simple curve, sufficiently smooth, one obtains

$$
\begin{aligned}
J^{\prime}(\Gamma) \theta= & \frac{1}{2} \int_{\Gamma}\left[A \varepsilon\left(u_{1}\right): \varepsilon\left(u_{1}\right)-A \varepsilon\left(u_{2}\right): \varepsilon\left(u_{2}\right)\right] \theta n_{1} \\
& -\int_{\Gamma} p\left[\operatorname{div}\left(u_{1}\right)-\operatorname{div}\left(u_{2}\right)\right] \theta n_{1} .
\end{aligned}
$$

In particular, if $\Gamma$ is the flat interface $\Gamma^{0}=(0,1) \times\left\{y_{0}\right\}$, the associated displacements are linear:

$$
u_{i}(x, y)=\left(0, \frac{g}{\lambda+2 \mu} y\right), \quad i=1,2 .
$$

This greatly simplifies the computations (for instance all the terms on $\Gamma_{0}$ involving curvature vanish) and one finds in (36) that $J^{\prime}\left(\Gamma_{0}\right) \theta=0$ for any $\theta$, i.e., the flat interface is a local extremum of the elastic energy functional $J$. We show below that the sign of the second derivative of $J$ with respect to the interface shape variation tells if the extremum is a minimum or a maximum of the energy functional.

\subsection{Second variation with respect to $\Gamma$}

With the notations of the previous section, the second derivative (with respect to the interface shape variation) of a volume integral is given by [29]

$$
\begin{aligned}
J_{1}^{\prime \prime}(\omega, \theta, \theta) & =\left(J_{1}^{\prime}\right)^{\prime}(\omega, \theta, \theta)-J_{1}^{\prime}(\omega)(\nabla \theta) \theta \\
& =\lim _{t \rightarrow 0} \frac{J_{1}^{\prime}(\omega+t \theta) \theta-J_{1}^{\prime}(\omega) \theta}{t}-J_{1}^{\prime}(\omega)(\nabla \theta) \theta .
\end{aligned}
$$

If $\omega$ and $\theta$ are sufficiently smooth, Simon [29] has shown that

$$
\begin{aligned}
J_{1}(\omega+t \theta)= & J_{1}(\omega)+t J_{1}^{\prime}(\omega) \theta \\
& +\frac{t^{2}}{2} J_{1}^{\prime \prime}(\omega, \theta, \theta)+o\left(t^{2}\|\theta\|\right) .
\end{aligned}
$$


For our objective functional in the form $J(\Gamma)=$ $-1 / 2 \int_{\Omega_{i}} A \varepsilon\left(u_{i}\right): \varepsilon\left(u_{i}\right)$, calculations similar to those presented in the Appendix show that at $\Gamma=\Gamma^{0}$

$$
J^{\prime \prime}\left(\Gamma_{0}, \theta, \theta\right)=-2 \int_{\Omega_{i}} A \varepsilon\left(u_{i}^{\prime}\right): \varepsilon\left(u_{i}^{\prime}\right),
$$

which is negative, since the elastic densities $A \varepsilon\left(u_{i}^{\prime}\right): \varepsilon\left(u_{i}^{\prime}\right)$ are quadratic and positive, and since the fields $u_{i}^{\prime}$ do not vanish identically. We can thus conclude that when $t$ is small enough

$$
\begin{aligned}
J\left(\Gamma_{0}+t \theta\right) & =J\left(\Gamma_{0}\right)+J^{\prime}\left(\Gamma_{0}\right) \theta+J^{\prime \prime}\left(\Gamma_{0}, \theta, \theta\right)+O\left(\|\theta\|^{2}\right) \\
& <J\left(\Gamma_{0}\right) .
\end{aligned}
$$

In other words, any variation away from the flat interface decreases the value of the total elastic energy, which demonstrates the instability of the flat interface. Had we disregarded the work due to the external force $g$ in equation (27), we would then have obtained an opposite sign (namely $\left.+\frac{1}{2} \int_{\Omega_{i}} A \varepsilon\left(u_{i}\right): \varepsilon\left(u_{i}\right)\right)$ for the relaxed energy, and thus stability would have been implied.

Finally if the boundary conditions at the bottom surface were different, we may ask the question regarding sensitivity of our conclusion. If, instead of imposing a zero displacement at the bottom surface, we apply a fixed load, as for the upper surface, the conclusion about stability is unchanged. Let us call $h$ the load, then one has to add to (27) the following term $-\int_{\Gamma_{B}} h n_{1} v_{1}$, then following exactly the same manipulations as with the last term in (27) we arrive at the same final conclusion (37). It would be interesting to investigate in the future more general boundary conditions in order to extract the generic conditions that trigger an instability.

\section{Discussion}

\subsection{Effect of external work on the calculation of the total energy}

A point which is worth mentioning is that in writing the total energy, we must include both the intrinsic elastic energy and the work due to the external forces. Carelessness (for example not including the work done by surface forces) would be penalized by a fallacious conclusion: the surface would be stable! A simple argument that the external forces must be included is that when we perform a variation of the energy with the respect to the displacement field we must arrive to the appropriate bulk (Lamé equation) and boundary conditions, otherwise, the considered equations would not fulfill mechanical equilibrium. This requirement has guided our considerations.

\subsection{Kinetics effects}

By comparing the final state to the initial one, we did not include, de facto, explicitly the notion of kinetics. It is quite clear that two mechanisms play a major role: dissolution and diffusion in the fluid interstices. This has been treated for the free surface case where it has been shown that both dissolution and diffusion may be limiting factors for rocks [21]. We are planning to include diffusion in the fluid layer, and due to the thin fluid layer, it is likely that diffusion should have a two dimensional character (i.e. like surface diffusion; the diffusion constant should then be renormalized by the fluid layer). We expect the spectrum for the surface fluctuation of diffusion to scale like $D \ell^{2} q^{4}$, where $\ell$ is the fluid thickness, and $D$ is the bulk diffusion constant in the liquid. By comparing to the usual diffusion limited spectrum $D q^{2}$, the effective diffusion should be lowered by a factor of the order of $q \ell \ll 1$ (wavelengths of stylolites are usually much bigger than the fluid thickness).

For example, it has been found in [21] for quartz and other rocks that the dissolution is the slowest mechanisms. Now due to the thin fluid layer, we expect diffusion to compete, if not to limit, the instability. We hope to report along these lines in the near future.

\subsection{Chemical potential considerations}

We translate now the energy calculations performed in the previous sections in terms of chemical potential, for the sake of future kinetic calculations. The chemical potential of a solid element at the interface is obtained from the energy change with respect to the interface variation. This corresponds to the cost in energy that is needed to create a bump (a volume element) on the interface. More precisely, let $E_{T}$ denote the sum of the elastic and surfaces energies, then the very definition of the change of the chemical potential is

$$
\Delta \mu_{T}=-\frac{\delta E_{T}}{\delta V}
$$

where $\delta$ denotes the functional derivative (derivative with respect to the interface shape variation). Since we limit ourselves to a one dimensional interface, the functional derivative corresponds to variation with respect to the interface profile $h(x)$. It follows that the added (or removed) volume element becomes an area element given by $\delta h d x$, where $d x$ is a fixed interval along the $x$ direction. Thus the chemical potential will be just proportional to $-\frac{\delta E_{T}}{\delta h}$. The surface energy per unit length along the $y$ direction reads

$$
E_{s}=\gamma \int\left(\left[1+\left(\frac{d h}{d x}\right)^{2}\right]^{1 / 2}-1\right) d x
$$

and its variation with respect to the profile $h(x)$ is given by

$$
\delta E_{s}=-\gamma \int \frac{d}{d x}\left(\frac{\frac{d h}{d x}}{\left[1+\left(\frac{d h}{d x}\right)^{2}\right]^{1 / 2}}\right) d x=-\gamma \int \kappa d x \delta h
$$


1 where we have set

$$
\kappa=-\frac{\frac{d^{2} h}{d x^{2}}}{\left[1+\left(\frac{d h}{d x}\right)^{2}\right]^{3 / 2}}
$$

which is nothing but the interface curvature. It follows that the contribution to the chemical potential from surface energy is given by

$$
\Delta \mu_{s}=-\frac{\delta E_{T}}{\delta V}=\gamma \kappa
$$

5 The contribution coming from elasticity is more subtle, since the elastic energy is defined in the bulk, while our wish is to define a surface chemical potential. It turns out that one may express the variation of the elastic energy with respect to the interface shape precisely as an integral over the surface, as written above for the surface energy in equation (40). The calculation is given in details in Section 4, and the desired result of the first variation is given by equation (36). In that section please note that $\delta h$ used above is equivalent to $\theta$ multiplied by the normal vector; actually only normal displacements cause a shape change. The change in chemical potential due to stress is thus given by

$$
\begin{aligned}
\Delta \mu_{e}= & -\frac{1}{2}\left[A \varepsilon\left(u_{1}\right): \varepsilon\left(u_{1}\right)-A \varepsilon\left(u_{2}\right): \varepsilon\left(u_{2}\right)\right] \\
& +p\left[\operatorname{div}\left(u_{1}\right)-\operatorname{div}\left(u_{2}\right)\right]
\end{aligned}
$$

where we recall that $u_{i}(i=1,2)$ is the displacement field in medium $i$ (see Fig. 3), $\varepsilon$ is the deformation (or strain) tensor given by $\varepsilon(u)=\left(\nabla u+\nabla u^{T}\right) / 2$, and $A$ is the fourth order tensor which enters Hooke's law, namely the stress tensor $\sigma$ is related to the deformation by $\sigma=A \varepsilon\left(A_{i j k l}=\right.$ $\lambda \delta_{i k} \delta_{j l}+\mu \delta_{i l} \delta_{j k}$ where $\lambda$ and $\mu$ are the Lamé coefficients). Finally $p$ is a Lagrange multiplier introduced in Section 4, and plays the role of a pressure like term of the thin fluid layer, but it must be solved for in a consistent manner, as we have seen in Section 4. We have seen that only in the linear regime $p$ coincides with the load $g$.

Note that if there was only one solid bounded by vacuum, or by a liquid, then the chemical potential would simply be given by

$$
\Delta \mu_{e}=\frac{1}{2} A \varepsilon(u): \varepsilon(u)
$$

as has been used in other contexts (see for example [14]).

Once the total chemical potential is obtained one can relate it to the kinetics of the interface. The most simple example is that the normal velocity is proportional to minus the chemical potential drop across the interface.

The surface evolution equation (at global equilibrium, as is the case in this problem) - or more precisely the normal velocity of the interface - vanishes if the chemical potential difference vanishes, or equivalently if the energy derivative with respect to the shape vanishes. The second variation of the energy with respect to the interface shape (which is computed in this paper) is proportional to the variation of the chemical potential $\Delta \mu$. It is the second variation of energy with respect to the shape that carries information on stability.

There are three major physical effects that drive the surface evolution: (i) if the interface is in contact with a reservoir of a liquid containing the molecules of the solid, and if one disregards diffusion (say if the attachement/detachment at the surface is the limiting mechanism), then the normal velocity is proportional to the chemical potential difference between two states (say the actual one and the initially flat one), this is the case treated in reference [14], (ii) if the surface dynamics evolves due to surface diffusion (as is probably the case for stylolites), then the surface velocity is proportional to the minus of the Laplacian of the chemical potential drop across the interface $\Delta \mu_{T}$. This is the case treated in AsaroTiller [3], and Yang and Srolovitz [34]. The conclusion about stability is the same in both cases, the difference is encoded in the proportionality pre-factor (which has the same sign in both cases) between the normal velocity and the second derivative of the energy with respect to the shape. (iii) Finally if diffusion in the bulk is included, then the normal velocity will be given by an integral equation, and the Kernel of the integral operator, is proportional to $\Delta \mu_{T}$ times a propagator (Green's function). The propagator expresses the fact that the dynamics becomes nonlocal (addition of mass at some point at the surface is felt by the molecules in the solution at a distant point-due to depletion-inducing thus a nonlocal self-interaction of the moving boundary). But in all the three cases, the instability is encoded in the sign of the second derivative of the energy (with respect to the interface shape). Of course the precise way the instability evolves later in time, depends on the kinetic mechanisms, but not the existence of the instability itself.

\subsection{Instability of solid-solid interfaces: application to stylolites}

Solid-solid interface roughening has also been studied, e.g. $[1,12]$, where the two solids have different elastic moduli. There, it was demonstrated that an instability can emerge only if the two solids have different material properties. This markedly differs from our situation where the instability does occur even when the two solids have identical elastic properties. This is traced back to the very difference of the two models: in our case it is the thin fluid layer that transmits the stresses and materializes the interface, while in [12], the interface notion looses its meaning if the two solids have identical material properties (Eqs. (22) and (23) in [12] implies that the elastic energy vanishes exactly for $\chi=1$, i.e. for identical material properties).

The present model considers a geometry which is close to that of a natural stylolite, where the interface separates two pieces of rock, and is a medium of dissolution in a fluid phase. Quantitative measurements on stylolite surfaces, using a high resolution profilometer, demonstrate 
that roughening do occur at all scales [27]. The interpretation of this observation is still controversial. It has been proposed that the roughening may be driven by a quenched noise initially present in the rock [6,27]. Here, we propose an alternative mechanism: stylolite might be inherently unstable, and the roughening could be driven by local gradients of strain energy. This interpretation is supported by the observation that stylolite do roughen even in very pure rocks such as chalk, where the amount of heterogeneities (quenched noise) is very low. However, further studies, together with laboratory experiments (mimicking the phenomenon) are needed before drawing more conclusive answers.

\section{Conclusion}

We have shown that a normal load on a solid-fluid-solid interface leads to an instability when using a boundary condition of transmission of the normal stress, but not the shear stress, across the interface. We have shown both explicitly (from linear theory with regard to the perturbation of a flat interface) and from a more general consideration (still within linear perturbation, but without having resort to an explicit solution of the elastic field) that the flat interface is unstable.

When comparing the final state corresponding to a modulated surface with the initial state having a flat surface, we have shown that the modulated surface has lower energy. Given this fact, and the fact related to the Asaro-Tiller-Grinfeld instability, it is appealing to speculate that this should be the case in an arbitrary geometry and arbitrary boundary conditions, provided that locally the considered moving interface possesses a non zero deviatoric stress component. A mathematical general proof is still lacking.

It must be kept in mind that the present study has introduced two simplifications. (i) The instability wavelength is small as compared with the lateral extent of the interface. This holds for natural interfaces that can be found in rocks, for example stylolites. If it occurs (in some special situation) that this is not the case, then one has to consider the role of lateral boundaries as well. (ii) We have considered a uniaxial stress and not a bi-axial one as occurs in realistic situations. Extensions to more general biaxial pre-stress would be interesting.

Finally, our study has focused on the birth of instability and on the lengthscales that are likely to grow first. Nonlinear effects should become decisive in the course of time as linear theory tells us that the amplitude should grow exponentially with time. How would the final state (if any) look like? How would coarsening (if any) occur, in that how fast is it? These question require a numerical study, and an appropriate way would be to make use of a phase-field model, like in [15].

We acknowledge financial support from the French ministry of research (PPF Dynamique des Systèmes Complexes). The support of Région Rhône Alpes (project Elasticité et Nanostruc- tures) and of the ANR project Géocarbone are also gratefully acknowledged.

\section{Appendix A: Proof of formula (36)}

We first recall that the local derivatives $u_{i}^{\prime}$ are $x$-periodic on $\Gamma_{V}$ and that $u_{1}^{\prime} \equiv 0$ on $\Gamma_{B}$. Taking the shape derivative (29) of the expression (27) of the objective functional, we obtain

$$
\begin{aligned}
J(\Gamma)^{\prime} \theta= & -\int_{\Omega_{i}} A \varepsilon\left(u_{i}\right): \varepsilon\left(u_{i}^{\prime}\right) \\
& -\frac{1}{2} \int_{\partial \Omega_{i}} A \varepsilon\left(u_{i}\right): \varepsilon\left(u_{i}\right) \theta n_{i} .
\end{aligned}
$$

Integrating by parts and using the fact that $\theta$ vanishes on the boundaries but on $\Gamma$ shows that

$$
\begin{aligned}
J(\Gamma)^{\prime} \theta= & -\int_{\Gamma} A \varepsilon\left(u_{i}\right) n_{i} u_{i}^{\prime}-\int_{\Gamma_{T}} g n_{2} u_{2}^{\prime} \\
& -\frac{1}{2} \int_{\Gamma} A \varepsilon\left(u_{i}\right): \varepsilon\left(u_{i}\right) \theta n_{i} .
\end{aligned}
$$

On the other hand, taking the shape derivative (30) of (28) yields

$$
J(\Gamma)^{\prime} \theta=\frac{-1}{2} \int_{\Gamma_{T}} g n_{2} u_{2}^{\prime}
$$

Combining the two previous expressions we obtain

$$
\begin{aligned}
J(\Gamma)^{\prime} \theta & =\int_{\Gamma} A \varepsilon\left(u_{i}\right) n_{i} u_{i}^{\prime}+\frac{1}{2} \int_{\Gamma} A \varepsilon\left(u_{i}\right): \varepsilon\left(u_{i}\right) \theta n_{i} \\
& =\int_{\Gamma} p n_{i} u_{i}^{\prime}+\frac{1}{2} \int_{\Gamma} A \varepsilon\left(u_{i}\right): \varepsilon\left(u_{i}\right) \theta n_{i} .
\end{aligned}
$$

To eliminate the local derivatives in the above equality, we take the shape derivative of the constraint on the displacements, which is conveniently rewritten

$$
\int_{\Gamma} u_{i} n_{i}=\int_{\Omega_{i}} \operatorname{div}\left(u_{i}\right)-\int_{\Gamma_{T}} u_{2} n_{2}=0,
$$

and obtain

$$
0=\int_{\Omega_{i}} \operatorname{div}\left(u_{i}^{\prime}\right)+\int_{\partial \Omega_{i}} \operatorname{div}\left(u_{i}\right) \theta n_{i}-\int_{\Gamma_{T}} u_{2}^{\prime} n_{2} .
$$

Integrating by parts the first term in the above expression, we arrive at

$$
\int_{\Omega_{i}} u_{i}^{\prime} n_{i}=-\int_{\Gamma} \operatorname{div}\left(u_{i}\right) \theta n_{i}
$$

Finally, injecting this equality in (45) proves (36).

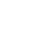
.

(n)

(1)




\section{References}

1. E. Jettestuen, L. Angheluta, J. Mathiesen, F. Renard, B. Jamtveit, Phys. Rev. Lett. 100, 096106 (2008)

2. F. Arthaud, M. Mattauer, Bull. Soc. Géol. Fr. 11, 738 (1969)

3. R.J. Asaro, W.A. Tiller, Met. Trans. 3, 1789 (1972)

4. R.G.C. Bathurst, Carbonate sediments and their diagenesis (Elsevier, Amsterdam, 1971)

5. J. Bisschop, D.K. Dysthe, Phys. Rev. Lett. 96, 146103 (2006)

6. A. Brouste, F. Renard, J.-P. Gratier, J. Schmittbuhl, J. Struc. Geol. 29, 422 (2007)

7. S.W.J. Den Brok, J. Morel, Geophys. Res. Lett. 28, 603 (2001)

8. H.V. Dunnington, J. Sed. Petrol. 24, 27 (1954)

9. D. Gal, A. Nur, E. Aharonov, Geophys. Res. Lett. 25, 1237 (1998)

10. J.P. Gratier, L. Muquet, R. Hassani, F. Renard, J. Struc. Geol. 27, 89 (2005)

11. M. Grinfeld, Sov. Phys. Dokl. 31, 831 (1986)

12. M.A. Grinfeld, J. Nonlinear Sci. 3, 35 (1993)

13. M.T. Heald, J. Geol. 63, 101 (1955)

14. K. Kassner, C. Misbah, Europhys. Lett. 28, 245 (1994)

15. K. Kassner, C. Misbah, Europhys. Lett. 46, 217 (1999)

16. K. Kassner, C. Misbah, J. Müller, J. Kappey, P. Kohlert, Phys. Rev. E 63, 036117 (2001)

17. R. Kerrich, Zentrabl. Geol. Paleontol. 5-6, 512 (1977)

18. D. Koehn, J. Arnold, A. Malthe-Srenssen, B. Jamtveit, Am. J. Sci. 303, 656 (2003)

19. D. Koehn, D.K. Dysthe, B. Jamtveit, Geochim. Cosmochim. Acta 68, 3317 (2004)

20. D.L. Landau, E.M. Lifchitz, Theory of elasticity, Butterworth Heinemann (Oxford, UK, 1999)
21. C. Misbah, F. Renard, J.-P. Gratier, K. Kassner, Geophys. Res. Lett. 31, L06618 (2004)

22. F. Murat, J. Simon, Sur le contrôle par un domaine géométrique, Rapport du Laboratoire d'Analyse Numérique, 189, 76015. Université de Paris 6, Paris (1976)

23. P. Nozieres, The grinfeld instability of stressed crystals, in NATO Ad-vanced Research Workshop on Spatio-Temporal Patterns in Nonequilibrium Complex Systems, edited by P.E. Cladis, P. Palffy-Muhoray, Vol. 21, 1994

24. W.C. Park, E.H. Schot, J. Sedimentary Petrology 38, 175 (1968)

25. L.B. Railsback, L.M. Andrews, J. Struc. Geol. 17, 911 (1995)

26. F. Renard, J. Schmittbuhl, J.P. Gratier, P. Meakin, E. Merino, J. Geophys. Res. 108, B03209 (2004)

27. J. Schmittbuhl, F. Renard, J.P. Gratier, R. Tous-saint, Phys. Rev. Lett. 93, 238501 (2004)

28. J. Simon, Second variations for domain optimization problems, in 4th Internationial conference on control of distributedparameter systems, International Series, edited by F. Kappel, K. Kunish, W. Schappacher, Birkhauser, Berlin, 1989

29. J. Simon, Differenciacion de problemas de con-torno respecto del dominio, lecture notes available at http://wwwlma. univ-bpclermont.fr/ simon/pagePubs . html\#vardomaine. Departamento de An'alisis Matem'atico, Universidad de Sevilla, Sevilla (1991)

30. H.C. Sorby, Proc. Royal Soc. London 12, 538 (1863)

31. D.J. Srolovitz, Acta Metall. 37, 621 (1989)

32. P.B. Stockdale, Stylolites: Their nature and origin, Ph.D. thesis, Indiana University Studies, 1922

33. R.H. Torii, S. Balibar, J. Low Temp. Phys. 89, 391 (1992)

34. W.H. Yang, D.J. Srolovitz, Phys. Rev. Lett. 71, 1593 (1993) 